\title{
Subgaleal Hematoma
}

National Cancer Institute

\section{Source}

National Cancer Institute. Subgaleal Hematoma. NCI Thesaurus. Code C116591.

A collection of blood between the scalp and the skull periosteum. 